\title{
Rituximab in ANCA-associated vasculitis presenting with severe acute kidney injury; a case report
} \author{
In-Ah Vanessa Park ${ }^{1}$ \\ ${ }^{1}$ The Robert Larner, M.D. College of Medicine, University of Vermont, Burlington, VT, USA \\ ${ }^{2}$ University of Port Harcourt Teaching Hospital, Port Harcourt, Nigeria \\ ${ }^{3}$ Nnamdi Azikiwe University Teaching Hospital, Nnewi, Nigeria \\ ${ }^{4}$ University of Nigeria Teaching Hospital, Enugu, Nigeria
}

Macaulay Onuigbo $^{* \mathbb{\oplus}}$, Julius Seok ${ }^{1}$, Ikenna Anyamene ${ }^{2}$, Fortunate Ejimone ${ }^{3}$, Chinenye Eze-Raphael ${ }^{4}$,

\section{A R T I C L E I N F O}

\section{Article Type:}

Case Report

\section{Article History:}

Received: 7 October 2018

Accepted: 14 December 2018

ePublished: 9 January 2019

\section{Keywords:}

Acute kidney injury, Anti-neutrophil

cytoplasmic antibody, Rituximab,

Vasculitis, Acute kidney injury,

Pulmonary-renal syndrome

\begin{abstract}
A B S T RA C T
Anti-neutrophil cytoplasmic antibody (ANCA)-associated vasculitis presenting with severe acute kidney injury (AKI), consistent with rapidly progressive glomerulonephritis portends significant renal morbidity with up to $20 \%-25 \%$ of patients reaching end stage renal disease within a few years after diagnosis. Nevertheless, a smaller proportion of patients require dialysis at presentation or within the first six months. There is still some debate as to the first line therapy of choice for such patients vis a vis the use of the newer agent rituximab versus the time-tested cyclophosphamide in combination with glucocorticoids. Our recent experience at The Robert Larner, M.D. College of Medicine, University of Vermont, Burlington, VT, USA in June 2018 allowed us to revisit this controversy.
\end{abstract}

Implication for health policy/practice/research/medical education:

In the last decade, there has been an increasing interest in the exact role of intravenous rituximab in the management of ANCAassociated vasculitis presenting with severe acute kidney injury. We describe a recent experience at The Robert Larner, M.D. College of Medicine, University of Vermont, Burlington, VT, USA that enabled us to revisit this controversy. There is indeed a place for rituximab in combination with glucocorticoids in these circumstances.

Please cite this paper as: Onuigbo M, Seok J, Anyamene I, Ejimone F, Eze-Raphael C, Vanessa Park IA. Rituximab in ANCAassociated vasculitis presenting with severe acute kidney injury; a case report. J Nephropharmacol. 2019;8(1):e13. DOI: 10.15171/ npj.2019.13

\section{Introduction}

In January 2011, the Boards of Directors of the American College of Rheumatology (ACR), the American Society of Nephrology (ASN), and the European League Against Rheumatism (EULAR) recommended that the name "Wegener's granulomatosis" be changed to "granulomatosis with polyangiitis", abbreviated as GPA (1). The natural history of similar Anti-neutrophil cytoplasmic antibody (ANCA)-associated vasculitis used to be progressive and fulminant with average survival rate of 5 months with mortality complicating either renal or respiratory failure (2). The administration of glucocorticoids improved survival to about a year, and the subsequent addition of cyclophosphamide in combination with glucocorticoids improved survival to ten years and going (3-5). In the last decade, the preponderance of cumulative evidence from various clinical trials has suggested that rituximab, a chimeric murine/human anti-CD20 monoclonal antibody that was first introduced to treat GPA in 2001 is an effective alternative to cyclophosphamide for both induction immunosuppressive therapy and for maintenance of remission (6). Efficacy, remission rates, relapse rates, and adverse effects are comparable between rituximab-based and cyclophosphamide-based therapies $(7,8)$. Nevertheless, younger patients with concerns about fertility may therefore opt for rituximab.

\section{Case Presentation}

An otherwise previously generally healthy 58 - year-old white male was evaluated as an inpatient in late May 2018 
for fever, fatigue, night sweats, cough and weight loss with imaging findings of bilateral multiple pulmonary nodules. A complete blood count revealed a leukocytosis of $12.0810^{9}$ per liter, an absolute neutrophilia of $9.3310^{9}$ per liter, mild anemia with hemoglobin of $11.8 \mathrm{~g} / \mathrm{dL}$ and platelet count of $38410^{9}$ per liter (141-377). However, the basic metabolic profile was normal, except for mild hyponatremia of $132 \mathrm{mmol} / \mathrm{L}$. Serum creatinine was 0.75 $\mathrm{mg} / \mathrm{dL}$. Aspartate aminotransferase (AST), and alanine aminotransferase (ALT), alkaline phosphatase and total bilirubin were all normal. C-reactive protein (CRP) was elevated at $254.1 \mathrm{mg} / \mathrm{L}(<10)$. The patient who has a history of recurrent sinus infections requiring antibiotic therapies was treated for an oral abscess in April 2018. Because of a persistent sore neck and additional suspicion of presence of thyroid nodules, he underwent imaging of his neck and this incidentally revealed abnormalities in both lung apices. A subsequent CT scan of the chest in the referral hospital then showed multiple areas of dense mass-like consolidation as well as some lymphadenopathy in the perihilar region and the patient was then transferred to our center for further evaluation.

Subsequent work up in our center demonstrated negative infectious disease work up including serologic HIV tests, blood cultures for bacteria, urine antigen tests for blastomyces, streptococcus pneumoniae and Legionella pneumophila. He had endoscopic bronchoscopy with a diagnostic bronchial alveolar lavage (BAL) of the right mid lung together with endo-bronchial ultrasoundguided fine needle aspirations from both the right upper lung and right mid lung lesions. The patient opted for discharge home after two days pending pathology and culture results. Thereafter, the BAL returned negative for fungal stains, fungal cultures and tests for acid-fast bacilli (AFB) including blood TB antigen value. The fineneedle aspiration lung biopsy showed atypical squamous metaplasia with marked acute and chronic inflammation and was negative for AFB and fungal stains and cultures.

He was however readmitted about ten days later with persistent worsening cough, bright red hemoptysis, about a teaspoonful of blood approximately every three hours, but without blood clots, more weight loss, fever, reduced urine output and darkening of the urine but without dysuria, frequency or flank pain. He had lost about 20 lbs. He described small rashes in his chest and leg and his appetite was poor.

In the emergency department, vitals were temperature $97.9^{\circ} \mathrm{F}$, pulse $91 /$ minutes, blood pressure $114 / 63 \mathrm{~mm} \mathrm{Hg}$, respiratory rate $19 / \mathrm{min}$ and oxygen saturation was $99 \%$ on room air. Hemoglobin was lower at $10.5 \mathrm{~g} / \mathrm{dL}$, white blood cell (WBC) was higher at $14.0110^{9}$ per liter, sodium was $132 \mathrm{mmol} / \mathrm{L}$, potassium $4.4 \mathrm{mmol} / \mathrm{L}$, bicarbonate 26 $\mathrm{mmol} / \mathrm{L}$ and serum creatinine had increased to $5.41 \mathrm{mg} /$ dL. Automated laboratory urinalysis revealed $3+$ blood, $1+$ protein, 1+ leukocyte esterase, 11-50 WBC per high power field (WBC/HPF), >50 red blood cell (RBC)/HPF and $<10$ hyaline casts/LPF.

Further physical examination revealed non-blanching, raised, non-tender petechial rash scattered over the abdomen, chest, the lower extremities, behind both elbows and the thighs, both ankles and the knees. We then completed a fresh bedside urine deposit microscopy on the first nephrology consult day. The urine was cloudy, amber colored but no overt hematuria. Urine deposit microscopy in the nephrology side laboratory revealed 5-10 WBC/ $\mathrm{HPF}$, 5-10 RBC/HPF including dysmorphic RBCs representing up to $30 \%$ of the RBC, and 1-3 granular casts/ LPF (Figure 1). This finding of dysmorphic RBCs, not reported in the automated laboratory urine microscopy raised the diagnostic possibility of a glomerulonephritis very significantly and we awaited the kidney biopsy result with great interest (2-9).

Albumin was $2.9 \mathrm{~g} / \mathrm{dL}$, phosphorus $5.0 \mathrm{mg} / \mathrm{dL}$ and $\mathrm{LDH}$ $787 \mathrm{U} / \mathrm{L}$. A random urine protein was $30 \mathrm{mg} / \mathrm{dL}$. Blood tests for ANA, C3 and C4 complements, anti-dsDNA, ASO, serum cryoglobulins, anti-GBM Ab, total CK, and TSH all returned negative or normal. Blood ANCA was positive at a titer of 1:40 (cytoplasmic speckled pattern) with negative myeloperoxidase $\mathrm{Ab}$ but positive proteinase $3 \mathrm{Ab}$ at 4.9 $\mathrm{U}(<0.4)$. Although serum kappa and lambda free light chains were elevated at $16.88 \mathrm{mg} / \mathrm{dL}$ and $11.29 \mathrm{mg} / \mathrm{dL}$, respectively, urine immunofixation was negative for free monoclonal light chains and serum immunofixation was negative for monoclonal immunoglobulins. The working diagnosis was AKI in the setting of a pulmonary-renal syndrome, and consistent with some form of a rapidly progressive glomerulonephritis $(8,9)$.

The kidney biopsy carried out on hospital day 1 revealed necrotizing vasculitis and segmental necrotizing glomerulonephritis with crescents. Immunofluorescence showed anti-C3 mesangial glomerular staining with no glomerular staining with the remaining antibodies. A simultaneous right trans-thoracic CT-guided needle core lung biopsy demonstrated alveolar tissue with acute necrotizing inflammation with giant cells. A thigh skin lesion biopsy showed necrotic epidermis, reactive

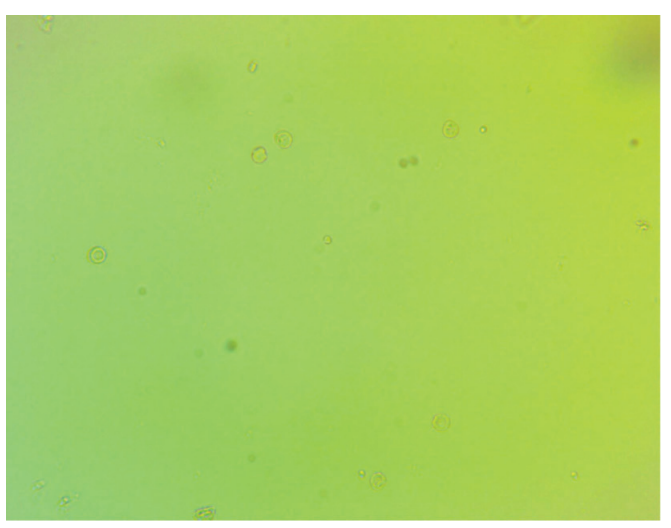

Figure 1. Bright field urine deposit microscopy showing 25\%-30\% dysmorphic RBCs consistent with a glomerulonephritis $(\times 400)$. 
changes but no evidence of vasculitis and negative immunofluorescence.

Immunosuppression was initiated starting with IV methyl-prednisone, 0.5 gm daily $\times 3$, on hospital day three with a plan to switch to oral prednisone $1 \mathrm{mg} / \mathrm{kg} / \mathrm{d}$ daily (60 mg daily) with a tapering protocol. Oral dapsone $100 \mathrm{mg}$ daily for pneumocystis jirovecii pneumonia (PJP) prophylaxis was started in place of trimethoprimsulfamethoxazole, because of the degree of renal failure, together with oral pantoprazole $40 \mathrm{mg}$ daily for GI prophylaxis. Amoxicillin-clavulanate 250-125 mg two times daily for 10 days was given for symptomatic sinusitis. On hospital day 6, after completing the three doses of methylprednisolone, following consultations between internal medicine, rheumatology and nephrology, IV rituximab $375 \mathrm{mg} / \mathrm{m}^{2}$ body surface area (BSA) weekly $\times 4$ was added to his induction immunosuppression regimen and he was to follow up on discharge with rheumatology. Each dose of rituximab infusion was preceded by premedication with oral diphenhydramine $50 \mathrm{mg}$, oral Acetaminophen $500 \mathrm{mg}$ PO and intravenous methylprednisolone $50 \mathrm{mg}$. Serum creatinine on discharge, on hospital day 7, was $3.84 \mathrm{mg} / \mathrm{dL}$, gross hemoptysis had resolved, and overall the patient felt much better with less fatigue.

About two weeks post-discharge, he was otherwise asymptomatic except for nasal congestion, some nonspecific cramping in the fingers, and the occasional swelling in the wrists and ankles. In July 2018, after completing the last fourth weekly dose of rituximab, a Westergren erythrocyte sedimentation rate (ESR) was normal at $1 \mathrm{~mm} / \mathrm{h}$ and CRP had normalized at $0.9 \mathrm{mg} / \mathrm{L}$. The latest serum creatinine was $1.32 \mathrm{mg} / \mathrm{dL}$, down from a peak level of $5.41 \mathrm{mg} / \mathrm{dL}$ (Figure 2). Prednisone taper will be monitored. He will continue monthly complete blood count, comprehensive metabolic panel, ESR and urinalysis and is due for an appointment in three months. The plan is to subsequently administer rituximab for maintenance therapy, 1 gm every 4 months, pending further review and as tolerated by the patient.

\section{Discussion}

Our patient had features consistent with granulomatosis with polyangiitis involving multiple systems including the lungs, the kidneys, the skin and the upper airways. He has so far responded very well to the combination glucocorticoid-rituximab regimen. There was no recourse to plasmapheresis since there was no life-threatening pulmonary hemorrhage, he did not need renal replacement therapy for renal failure and serum antiGBM Ab was negative (6). His kidney function is very much improved, even though not fully back to baseline (Figure 2). This is yet another case to support a first-line role for rituximab in combination with glucocorticoids in the management of ANCA-associated vasculitis with severe renal involvement (6-9). Adverse drug effects have

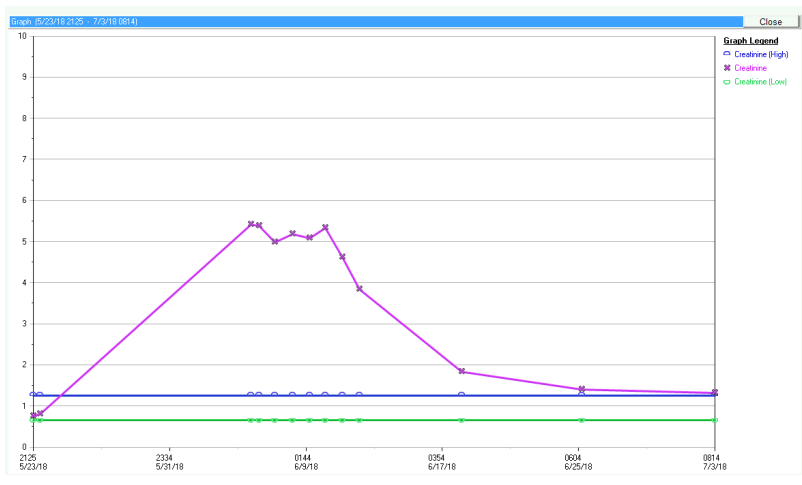

Figure 2. Serum creatinine trajectory May to July 2018, following rituximab-glucocorticoid combination induction immunosuppression regimen.

been otherwise minimal and the regimen has been well tolerated. The plan for a rituximab-based maintenance immunosuppression regimen would be revisited at his next appointment in three months (9). In the interim, he would continue to get monthly blood draws for complete blood count, comprehensive metabolic profile and urinalysis.

Notably, a just published 2018 review of the role of rituximab in ANCA-associated vasculitis with severe acute kidney injury had concluded that in such cases, cyclophosphamide should be the preferred first-line therapeutic agent (9). Nevertheless, arguably, the published data from the two largest randomized controlled trials, the RAVE and RITUXVAS trials, demonstrated that rituximab, a B cell-depleting agent, is as effective as cyclophosphamide for induction of remission in patients with newly diagnosed granulomatosis with polyangiitis and microscopic polyangiitis $(8,9)$. The jury is still out on this ongoing controversy as to the equivalence, inferiority or otherwise of rituximab-based therapies versus cyclophosphamide-based therapies (10).

\section{Authors' contribution}

Conception of the project; MACO. Data acquisition; MACO, JS, IVP. Literature review; MACO, JS, IA, FE, CE and IVP. Drafting the manuscript; MACO. Final approval of the article; MACO, JS, IA, FE, CE and IVP.

\section{Conflicts of interest}

The authors report no conflicts of interest. The authors alone are responsible for the content and writing of the article.

\section{Ethical considerations}

Ethical issues (including plagiarism, data fabrication, double publication) have been completely observed by the authors. The patient has given his informed consent regarding this case report.

\section{Funding/Support}

None. 


\section{References}

1. Falk RJ, Gross WL, Guillevin L, Hoffman GS, Jayne DR, Jennette JC, et al. Granulomatosis with polyangiitis (Wegener's): an alternative name for Wegener's granulomatosis. Arthritis Rheum. 2011;63:863-4. doi: 10.1002/art.30286.

2. Walton EW. Giant-cell granuloma of the respiratory tract (Wegener's Granulomatosis). Br Med J. 1958;2:265-70.

3. Fauci A, Wolff S. Wegener's granulomatosis: studies in eighteen patients and a review of the literature. Medicine (Baltimore). 1973;52:535-61.

4. Novack ST, Pearson CM. Cyclophosphamide therapy in Wegener's Granulomatosis. N Engl J Med. 1971;284:938-42.

5. Fauci AS, Haynes BF, Katz P, Wolff SM. Wegener's granulomatosis: prospective clinical and therapeutic experience with 85 patients for 21 years. Ann Intern Med. 1983;98:76-85.

6. Hassan RI, Gaffo AL. Rituximab in ANCA-Associated
Vasculitis. Curr Rheumatol Rep. 2017;19:6. doi: 10.1007/ s11926-017-0632-1.

7. Stone JH, Merkel PA, Spiera R, Seo P, Langford CA, Hoffman GS, et al. Rituximab versus cyclophosphamide for ANCAassociated vasculitis. N Engl J Med 2010;363:221232. doi: 10.1056/NEJMoa0909905.

8. Jones RB, Tervaert JW, Hauser T, Luqmani R, Morgan $\mathrm{MD}$, Peh CA, et al. Rituximab versus cyclophosphamide in ANCA-associated renal vasculitis. N Engl J Med 2010;363:211-220. doi 10.1056/NEJMoa0909169.

9. Salvadori M, Tsalouchos A. Antineutrophil cytoplasmic antibody associated vasculitides with renal involvement: Open challenges in the remission induction therapy. World J Nephrol. 2018;7(3):71-83. doi: 10.5527/wjn.v7.i3.71.

10. Moiseev S, Novikov P, Jayne D, Mukhin N. End-stage renal disease in ANCA-associated vasculitis. Nephrol Dial Transplant 2017;32:248-53. doi: 10.1093/ndt/gfw046.

Copyright (c) 2019 The Author(s); Published by Society of Diabetic Nephropathy Prevention. This is an open-access article distributed under the terms of the Creative Commons Attribution License (http://creativecommons.org/licenses/by/4.0), which permits unrestricted use, distribution, and reproduction in any medium, provided the original work is properly cited. 